\title{
ESTIMACIÓN DISTRIBUIDA DE SISTEMAS LTI BASADA EN CONFIANZA
}

\author{
A. Rodríguez del Nozal \\ Departamento de Ingeniería Eléctrica, Universidad de Sevilla, alvarordn@hotmail.com \\ L. Orihuela, P. Millán \\ Departamento de Ingeniería, Universidad Loyola Andalucía, \{dorihuela,pmillan\}@uloyola.es
}

\section{Resumen}

\begin{abstract}
Este artículo trata el problema de la estimación distribuida del estado de una planta por parte un conjunto de agentes. El objetivo de cada agente es el de reconstruir el estado completo a partir de sus propias medidas y la información intercambiada con el resto de agentes. Se considera la existencia de eventos aleatorios, por ejemplo ciberataques o fallos en los sensores, que pueden afectar a los agentes y propagarse a través de los vecinos. El artículo introduce un diseño robusto de observador y un algoritmo de ponderación para combatir este problema.
\end{abstract}

Palabras clave: Estimación distribuida. Sistemas lineales invariantes en el tiempo. Ciberataques.

\section{Introducción}

En determinadas aplicaciones se hace necesario estimar el valor de las variables de estado de un cierto sistema para llevar a cabo estrategias de control o monitorización sobre el mismo. La estimación de estas variables es lo que comúnmente se conoce como estimación de estado. Convencionalmente, tanto la estimación como el control de un sistema o planta se lleva a cabo de forma centralizada. Esto es, la información de todas las medidas de los sensores se transmite periódicamente a un mismo dispositivo que implementa los algoritmos necesarios para calcular en tiempo real el estado del sistema y/o las señales de control que deben aplicar los actuadores de la planta. Sin embargo, cuando aumenta el tamaño y la complejidad del sistema a controlar, o bien cuando se introducen redes de comunicación compartidas o inalámbricas, las probabilidades de fallos o ataques en los dispositivos aumentan y los sistemas centralizados se vuelven muy vulnerables. Ante estas circunstancias, se desarrollan los sistemas de control basados en red [5] o NCS (del inglés, Networked Control Systems), en los que se comienzan a implementar nuevas técnicas de estimación y control distribuido, en las que un grupo de dispositivos inteligentes realizan medidas del estado y las intercambian para llevar a cabo acciones de forma coordinada [4].

Si bien el enfoque distribuido de la estimación de estado ofrece importantes ventajas: reducir costes, aumentar la escalabilidad o mejorar la flexibilidad [12], no es menos cierto que el diseño de los algoritmos se vuelve más complejo. En particular, es necesario considerar que en general las medidas de los sensores van a ser recolectadas por distintos agentes espacialmente dispersos y con capacidad de comunicar información sólo a otros agentes dentro de su "vecindad".

El problema de la estimación distribuida del estado de una planta ha sido resuelto desde diferentes perspectivas en los último años. Quizás, el estimador más conocido sea el filtro de Kalman distribuido [10, 11]. Esta estrategia ha sido desarrollada en multitud de trabajos para dar solución a diferentes aplicaciones como pueden ser el procesamiento de señales [9], la visión artificial [13] o el campo de la óptica [3].

Es interesante destacar también otros estimadores utilizados para resolver el mismo problema. Es el caso de [1] y [7] dónde se utiliza una estructura de observador que, mediante una transformación del espacio de estados, permite identificar la parte observable y no observable de cada agente. Así, cada agente es capaz de reconstruir su parte observable mediante las medidas locales del estado y, por otro lado, la parte del estado no observable a través del intercambio de información con sus vecinos.

Finalmente, cuando nos centramos en el problema de estimación distribuida, muchos autores consideran la aparición de eventos aleatorios como pueden ser fallos en comunicaciones [8], cambios de topologías [14] o la posible inyección de información maliciosa derivada de ciberataques [6].

Este artículo trata de introducir una solución al problema de estimación distribuida del estado de una planta en un contexto en el que los agentes pueden sufrir fallos o verse afectados por entidades maliciosas que corrompan las medidas. Para ello, el artículo considera un caso sencillo con sólo tres agentes y presenta un diseño robusto de estimador 
y un algoritmo para limitar el efecto de dichos fallos o ataques en la estimación del estado por parte del grupo de agentes.

El artículo esta organizado de la siguiente manera. La Sección 2 introduce información necesaria para entender y plantear el resto del problema. En la Sección 3 se muestra la estructura de observador considerada así como las hipótesis necesarias para resolver el problema. La Sección 4 aborda el algoritmo . El procedimiento de diseño y el algoritmo ideado para rechazar la infromación maliciosa es presentada en la Sección 5. Finalmente en las secciones 6 y 7 se presntan varias simulaciones y las conclusiones del problema.

\section{Información del problema y preliminares}

Considere un sistema discreto, lineal, autónomo e invariante en el tiempo que está siendo observado por un conjunto de tres agentes. La representación en el espacio de estados del sistema y las medidas tomadas por los agentes vienen dadas por las siguientes ecuaciones:

$$
\begin{aligned}
x^{+} & =A x, \\
y_{i} & =C_{i} x,
\end{aligned}
$$

dónde $x \in \mathbb{R}^{n}$ es el estado, $A \in \mathbb{R}^{n \times n}$ es la matriz del sistema y $C_{i} \in \mathbb{R}^{m_{i} \times n}$ y $y_{i} \in \mathbb{R}^{m_{1}}$ representan respectivamente la matriz de salida y la medida de un agente $i \in\{1,2,3\}$.

Considere además que los agentes se comunican entre ellos de acuerdo a la topología mostrada en la Figura 1.

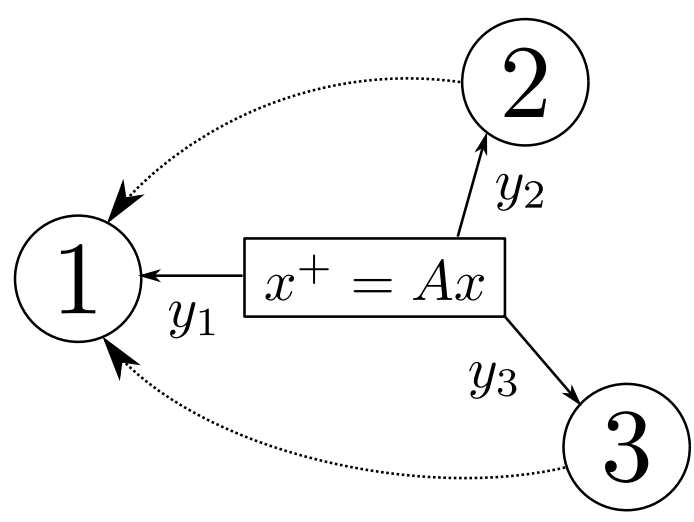

Figura 1: Esquemático del sistema considerado.

Este artículo considera un caso sencillo dentro del problema general de estimación distribuida tolerante a fallos o ataques. El problema estudiado se centra en analizar el diseño y el desempeño de un agente genérico (Agente 1) en su tarea de estimar el estado completo de un sistema a partir de sus propias medidas de la planta y de la información recibida a través de otros dos agentes agentes. Para ello, se propone un diseño que considera la existencia de eventos aleatorios en los que el Agente 1 puede recibir información corrupta de sus vecinos derivada de fallos en los sensores o de ciberataques.

La finalidad del artículo es el de introducir un algoritmo capaz de operar ante estos eventos de forma robusta reduciendo su efecto en la estimación del estado de la planta.

Para resolver el problema, se hará uso de una estructura de observador previamente presentada por los autores [1], [2], [7]. Dicha estructura se basa en una transformación del espacio de estados a través del cambio de variable $\xi_{1} \triangleq T_{1} x$ :

$$
\begin{aligned}
& \xi_{1}^{+}=T_{1}^{\top} A T_{1} \xi_{1}=\left[\begin{array}{cc}
\bar{V}_{1}^{\top} A \bar{V}_{1} & \bar{V}_{1}^{\top} A V_{1} \\
0 & V_{1}^{\top} A V_{1}
\end{array}\right] \xi_{1}, \\
& y_{1}=C_{1} T_{1} \xi=\left[\begin{array}{ll}
0 & V_{1} C_{1}
\end{array}\right] \xi,
\end{aligned}
$$

dónde $\bar{V}_{1} \in \mathbb{R}^{n \times n \bar{o}_{1}}$ esta compuesto por $n \bar{o}_{1}$ vectores columnas en $\mathbb{R}^{n}$ que conforman el espacio no observable del sistema por el Agente 1. A su vez, $V_{1} \in \mathbb{R}^{n \times n o_{1}}$ esta compuesto por $n o_{1}$ vectores columnas en $\mathbb{R}^{n}$ que conforman el espacio observable del sistema por el Agente 1, es decir, el espacio observable correspondiente a la pareja $\left(C_{1}, A\right)$.

Dado que ambas matrices se constituyen por vectores generadores ortonormales entre sí y a su vez generan dos subespacios ortogonales (subespacios observable y no observable), se cumple que:

$$
\begin{gathered}
\bar{V}_{1}^{\top} \bar{V}_{1}=I_{n \bar{o}_{1}}, \quad V_{1}^{\top} V_{1}=I_{n o_{1}}, \\
\bar{V}_{1}^{\top} V_{1}=0_{n \bar{o}_{1} \times n o_{1}}, \quad V_{1}^{\top} \bar{V}_{1}=0_{n o_{1} \times n \bar{o}_{1}} .
\end{gathered}
$$

\section{Estructura de observador propuesto y objetivo de diseño}

Esta sección presenta la estructura de observador considerada. Dicha estructura es similar a la presentada en [1], aunque añadiendo los pesos $c_{1, j} \in \mathbb{R}$, cuyo significado se discutirá en las siguientes secciones y cuyo fin consiste en permitir a los observadores operar de forma rubusta ante información corrupta:

$$
\begin{aligned}
\widehat{x}_{1}^{+}= & A \widehat{x}_{1}+V_{1} L_{1}\left(y_{1}-\widehat{y}_{1}\right) \\
& +\sum_{j \in \mathcal{N}_{1}} c_{1, j} \bar{V}_{1} N_{1, j} \bar{V}_{1}^{\top}\left(\hat{x}_{j}-\hat{x}_{1}\right),
\end{aligned}
$$

dónde $\widehat{x}_{1}$ es la estimación del estado de la planta $x$ obtenida por el Agente 1 y $L_{1}$ y $N_{1, j}$ son, respectivamente, un observador local y unas ganancias de consenso que deben ser diseñadas. Definimos 
$\mathcal{N}_{1}$ como el vecindario del Agente 1, en este caso, los agentes 2 y 3 (ver Figura 1).

Merece la pena aclarar que la estructura esta compuesta por tres términos: un término correspondiente al modelo del sistema, un segundo término que permite corregir la estimación a partir de las medidas obtenidas de la planta, y que tiene influencia sobre el subespacio observable del Agente 1, y finalmente un tercer término que corrige la estimación en el subespacio no observable de este mismo agente, gracias a la información proporcionada por sus vecinos.

Realizaremos la hipótesis durante todo el artículo de que el sistema (1) es colectivamente observable por el Agente 1, es decir, la pareja $\left(\bar{C}_{1}, A\right)$ es observable, dónde $\bar{C}_{1}$ es una matriz que apila la matriz de salida del Agente 1 y las de su vecindario: $\bar{C}_{1}^{\top}=\left[\begin{array}{lll}C_{1}^{\top} & C_{2}^{\top} & C_{3}^{\top}\end{array}\right]$.

Del mismo modo, ya que no es el foco del problema que se estudia en este artículo, se asume que los Agentes 2 y 3 pueden observar el estado completo del sistema (parejas $\left(C_{2}, A\right)$ y $\left(C_{3}, A\right)$ observables) y operan de acuerdo a cualquier diseño convencional de observador (e.j. observador de Luenberguer) que garantiza su convergencia al estado de la planta en ausencia de fallos o ataques.

\subsection{Diseño y operación distribuida}

El procedimiento de estimación del estado de la planta (1) por el Agente 1 se estructura en los siguientes tres pasos:

Fase de inicialización: En esta fase, el agente identifica su espacio observable a través de la dinámica del sistema y su matriz de salida. A partir de esta información, es capaz de construir la matrices $V_{1}$ y $\bar{V}_{1}$. Estas matrices servirán para definir la estructura de observación. El agente también identifica que vecinos constituyen su vecindario. Estas construcciones puede hacerse de acuerdo a [7].

Selección de las ganancias del observador: En esta fase, el Agente 1 diseña sus ganancias $\left(L_{1}, N_{1,2}, N_{1,3}\right)$ con el fin de garantizar la estabilidad y robustez del sistema.

Fase de operación: En la fase de operación los agentes toman medidas e intercambian la información necesaria para llevar a cabo la estimación del estado de la planta. En esta fase entra en juego el procedimiento de estimación basado en confianza que se propone para el Agente 1.

\section{Análisis de la estimación basada en confianza}

Como se discute en [6], el término confianza puede ser encontrado en la literatura de diversas maneras y con diferentes significados. En este artículo, definimos confianza en un agente como la fiabilidad que se puede otorgar a la información provista por él.

En el caso particular que se analiza en este artículo, el funcionamiento normal del proceso de estimación resulta en que en cada instante de muestreo los agentes 2 y 3 envían sus estimaciones del estado de la planta al Agente 1. Se propone aprovechar que el Agente 1 puede observar los patrones de comportamiento de sus vecinos e identificar cuándo se producen cambios significativos en ellos.

Con el fin de medir la confianza de un agente en otro definimos el parámetro $e_{i, j}$ como la diferencia entre la estimación del estado de la planta por dos agentes $i$ y $j$ proyectada sobre el espacio no observable del primero, esto es, $e_{i, j}=\bar{V}_{i}^{\top}\left(\hat{x}_{i}-\hat{x}_{j}\right)$. Finalmente, definimos $d_{Q}\left(e_{i, j}\right)$ como la distancia entre ambas estimaciones ponderadas mediante la matriz $Q$ :

$$
d_{Q}\left(e_{i, j}\right)=\sqrt{e_{i, j}^{\top} Q e_{i, j}}
$$

Así, mediante un escalar, podemos cuantificar la confianza del Agente 1 en cada uno de sus vecinos, teniendo en cuenta el subespacio no observable por el primero. En los siguientes párrafos, se describen varias zonas de confianza atendiendo a los valores tomados por $d_{Q}\left(e_{1, j}\right)$.

Considere una situación inicial en la que las ganancias $L_{1}, N_{1,2}$ y $N_{1,3}$ han sido diseñadas para garantizar la estabilidad en la estimación del Agente 1 bajo un funcionamiento normal del sistema, es decir, sin considerar fallos o ataques sobre los agentes. Suponga también un valor inicial de $c_{1,2}=c_{1,3}=1$. Bajo este escenario diremos que las distancias entre estimaciones se encuentran en la región de confianza $\mathcal{R} \triangleq\left\{d_{Q}\left(e_{1, j}\right): d_{Q}\left(e_{1, j}\right)<\right.$ $\left.r_{1, j}\right\}$.

Imagine ahora la situación en la que el Agente 1 comienza a recibir información de uno de sus vecinos que no sigue los patrones de comportamiento observados hasta entonces. Este hecho ocasionará que el valor de $d_{Q}\left(e_{1, j}\right)$ abandone la región de confianza afectando a la estabilidad en la estimación. Entonces, la distancia entre estimaciones pasará a estar en la región de alerta, $\mathcal{A} \triangleq\left\{d_{Q}\left(e_{1, j}\right): r_{1, j} \leq\right.$ $\left.d_{Q}\left(e_{1, j}\right) \leq a_{1, j}\right\}$.

En la zona de alerta se considera que los valores de $d_{Q}\left(e_{1, j}\right)$ no son suficientemente altos y que aún 
se puede tener un cierto grado de confianza en el Agente j. Sin embargo, dicho grado de confianza debe disminuir el impacto de sus información sobre la estimación del estado hecha por el Agente 1. Para ello, el Agente 1 disminuirá el valor de $c_{1, j}$.

Si el valor de $d_{Q}\left(e_{1, j}\right)$ continua creciendo, diremos que el valor de $d_{Q}\left(e_{1, j}\right)$ entra en la región de desconfianza, $\mathcal{D} \triangleq\left\{d_{Q}\left(e_{1, j}\right): a_{1, j} \leq d_{Q}\left(e_{1, j}\right)\right\}$. En esta región se considera que la información recibida se es totalmente corrupta y por lo tanto no es útil para el Agente 1, tendiendo el peso $c_{1, j}$ a un valor muy cercano a cero. En este caso, se vuelve a llevar a cabo la fase de inicialización descrita en la Sección 3.1, pero esta vez, sin tener en cuenta al agente que provee información errónea. Tenga en cuenta que para excluir a dicho agente es necesario garantizar la observabilidad colectiva de la planta por parte del Agente 1.

En caso de que el agente afectado consiga recuperarse, podrá volver a entrar en el proceso de estimación siempre y cuando disminuya el valor de $d_{Q}\left(e_{1, j}\right)$ de tal manera que se pueda volver a confiar en él, es decir, cuando alcance nuevamente la región de confianza, $\mathcal{R}$.

\section{Procedimiento de estimación basada en confianza}

La sección anterior nos ha permitido definir las tres diferentes zonas en las que $d_{Q}\left(e_{1, j}\right)$ puede situarse. Esta sección propone un diseño robusto de observador así como un algoritmo que permita modificar los pesos $c_{1, j}$ con el fin de minimizar el efecto de información corrupta.

\subsection{Diseño robusto del observador}

Después de llevar a cabo la fase de inicialización descrita en la Sección 3.1, es necesario diseñar las ganancias $L_{1}, N_{1,2}$ y $N_{1,3}$ de forma adecuada. Con el fin de lograr ese objetivo, esta sección propone un diseño robusto de dichas ganancias atendiendo a todos los valores posibles que puede tomar los pesos $c_{1,2}$ y $c_{1,3}$. Para ello, es necesario conocer la evolución de la dinámica del error de estimación.

\subsubsection{Dinámica del error de estimación}

En primer lugar, definamos el error de estimación del Agente 1 como la diferencia entre el estado real de la planta y la estimación hecha por dicho agente: $e_{1}=x-\hat{x}_{1}$. Si aplicamos la transformación dada por la matriz $T_{1}$ al error de estimación, es posible obtener:

$$
\epsilon_{1}=\left[\begin{array}{l}
\bar{\varepsilon}_{1} \\
\varepsilon_{1}
\end{array}\right]=\left[\begin{array}{ll}
\bar{V}_{1}^{\top} & e_{1} \\
V_{1}^{\top} & e_{1}
\end{array}\right] .
$$

A partir de las expresiones del error expuestas y de la estructura de observador definida en (4), es posible calcular la evolución del error de estimación como:

$$
\begin{aligned}
e_{1}^{+} & =x^{+}-\hat{x}_{1}^{+} \\
& =A x-A \widehat{x}_{1}-V_{1} L_{1} C_{1}\left(x-\widehat{x}_{1}\right) \\
& -\sum_{j \in \mathcal{N}_{1}} c_{1, j} \bar{V}_{1} N_{1, j} \bar{V}_{1}^{\top}\left(\widehat{x}_{j}-\widehat{x}_{1}\right) \\
& =\left(A-V_{1} L_{1} C_{1}\right) e_{1} \\
& -\sum_{j \in \mathcal{N}_{1}} c_{1, j} \bar{V}_{1} N_{1, j} \bar{V}_{1}^{\top}\left(\widehat{x}_{j}-x+x-\widehat{x}_{1}\right) \\
& =\left(A-V_{1} L_{1} C_{1}\right) e_{1} \\
& -\sum_{j \in \mathcal{N}_{1}} c_{1, j} \bar{V}_{1} N_{1, j} \bar{V}_{1}^{\top}\left(e_{1}-e_{j}\right) .
\end{aligned}
$$

Aplicando la transformación introducida en la Sección 2, y suponiendo que el error de estimación de los vecinos del Agente 1 tienden a cero con el tiempo, es posible obtener el error de estimación de los estados observables y no observables por el agente:

$$
\begin{aligned}
{\left[\begin{array}{l}
\bar{\varepsilon}_{1} \\
\varepsilon_{1}
\end{array}\right]^{+}=} & {\left[\begin{array}{cc}
\bar{V}_{1}^{\top} A \bar{V}_{1}-\sum_{j \in \mathcal{N}_{1}} c_{1, j} N_{1, j} & \ldots \\
0 & \\
\bar{V}_{1}^{\top} A V_{1} \\
& \cdots V_{1}^{\top} A V_{1}-L_{1} C_{1} V_{1}
\end{array}\right]\left[\begin{array}{l}
\bar{\varepsilon}_{1} \\
\varepsilon_{1}
\end{array}\right] . }
\end{aligned}
$$

De esta matriz con forma de cascada es deducible que la dinámica del error de estimación viene dada por los autovalores de las matrices situadas en las diagonal, que, a su vez, fijan el ratio de convergencia del estimador en sus partes observable y no observable. Así, es fácil ver que si las matrices:

$$
\begin{aligned}
& D=V_{1}^{\top} A V_{1}-L_{1} C_{1} V_{1}, \\
& \bar{D}=\bar{V}_{1}^{\top} A \bar{V}_{1}-\sum_{j \in \mathcal{N}_{1}} c_{1, j} N_{1, j}
\end{aligned}
$$

presentan un radio espectral menor que uno, entonces la estimación del estado de la planta por parte del Agente 1 tenderá asintóticamente al estado de ésta.

\subsubsection{Método de diseño propuesto}

A partir de la expresión (5) es posible encontrar una ganancia $L_{1}$ que permita fijar los polos de $D$ de forma arbitraria. Sin embargo, en el caso de la expresión (6) dos conjuntos de variables aparecen en escena. Por un lado, el diseño de las ganancias de consenso $N_{1,2}$ y $N_{1,3} \mathrm{y}$, por otro lado, el diseño de los pesos $c_{1,2}$ y $c_{1,3}$.

Como se desarrolló en la Sección 4 el valor de los pesos $c_{1,2}$ y $c_{1,3}$ variará entre un valor máximo 
igual a 1, para la región de confianza, y un valor entre 1 y un mínimo $c_{m i n}$ para la región de alerta. Una vez que la distancia $d_{Q}\left(e_{1, j}\right)$ entre en la región de desconfianza, se genera una reestructuración del observador (4) no contemplando las medidas provistas por el Agente j que suministra información corrupta (es decir, se considera $c_{1 j}=0$ )

Así, este artículo propone un diseño de las matrices $N_{1,2}$ y $N_{1,3}$ tal que las siguientes matrices tengan un radio espectral menor que uno:

$$
\begin{aligned}
& M_{1}=\bar{V}_{1}^{\top} A \bar{V}_{1}-N_{1,2}-N_{1,3}, \\
& M_{2}=\bar{V}_{1}^{\top} A \bar{V}_{1}-c_{\min } N_{1,2}-c_{m i n} N_{1,3}, \\
& M_{3}=\bar{V}_{1}^{\top} A \bar{V}_{1}-N_{1,2}-c_{m i n} N_{1,3}, \\
& M_{4}=\bar{V}_{1}^{\top} A \bar{V}_{1}-c_{m i n} N_{1,2}-N_{1,3} .
\end{aligned}
$$

Observe que las matrices expuestas arriba son una particularización de (6) para los valores extremos de los pesos $c_{i, 1}$ y $c_{i, 2}$. Así, dada la linealidad del problema, es fácilmente deducible que, si se cumple el criterio de estabilidad para los casos extremos, también lo hará para valores de $c_{1,2}$ y $c_{1,3}$ comprendidos entre 1 y $c_{m i n}$.

Además, es fácil darse cuenta que las expresiones de las matrices $M_{1}, M_{2}, M_{3}$ y $M_{4}$ pueden ser reescritas de forma matricial de las siguiente manera:

$$
\begin{aligned}
& M_{1}=\bar{V}_{1}^{\top} A \bar{V}_{1}-\left[\begin{array}{ll}
I_{n \bar{o}_{i}} & I_{n \bar{o}_{i}}
\end{array}\right]\left[\begin{array}{l}
N_{1,2} \\
N_{1,3}
\end{array}\right], \\
& M_{2}=\bar{V}_{1}^{\top} A \bar{V}_{1}-c_{\min }\left[\begin{array}{ll}
I_{n \bar{o}_{i}} & I_{n \bar{o}_{i}}
\end{array}\right]\left[\begin{array}{l}
N_{1,2} \\
N_{1,3}
\end{array}\right], \\
& M_{3}=\bar{V}_{1}^{\top} A \bar{V}_{1}-\left[\begin{array}{ll}
I_{n \bar{o}_{i}} & c_{m i n} I_{n \bar{o}_{i}}
\end{array}\right]\left[\begin{array}{l}
N_{1,2} \\
N_{1,3}
\end{array}\right], \\
& M_{4}=\bar{V}_{1}^{\top} A \bar{V}_{1}-\left[\begin{array}{ll}
c_{m i n} I_{n \bar{o}_{i}} & I_{n \bar{o}_{i}}
\end{array}\right]\left[\begin{array}{l}
N_{1,2} \\
N_{1,3}
\end{array}\right] .
\end{aligned}
$$

Así, el diseño se reduce a obtener el valor de la matriz $\left[\begin{array}{l}N_{1,2} \\ N_{1,3}\end{array}\right]$ que haga que $M_{1}, M_{2}, M_{3}$ y $M_{4}$ tengan radio espectral menor que uno.

\subsubsection{Actualización de la confianza}

Cuando sucede que uno de los agentes esta enviando información corrupta y el valor de la distancia del error de estimación se encuentra dentro de la zona de alerta, parece lógico asignar un peso $c_{1, j}$ inversamente proporcional al valor de $d_{Q}\left(e_{1, j}\right)$. En este artículo se propone la siguiente actualización de los pesos:

$$
c_{1, j}=\left\{\begin{array}{ccc}
\frac{r_{1, j}}{d_{Q}\left(e_{1, j}\right)} & \text { if } & d_{Q}\left(e_{1, j}\right)>r_{1, j} \\
1 & \text { if } & d_{Q}\left(e_{1, j}\right) \leq r_{1, j}
\end{array},\right.
$$

dónde $r_{1, j}$ es el límite que para $d_{Q}\left(e_{1, j}\right)$ que lo hace entrar en la región de alerta $\mathcal{A}$.

\section{Ejemplos de simulación}

Con el fin de mostrar la robustez del algoritmo introducido se llevarán a cabo varias simulaciones. Para ello considere el siguiente sistema y matrices de salida:

$$
\begin{gathered}
A=\left[\begin{array}{ccc}
1.005 & 0 & 0 \\
0 & 0.9954 & -0.08757 \\
0 & 0.1248 & 0.9945
\end{array}\right], \\
C_{1}=\left[\begin{array}{l}
1 \\
0 \\
0
\end{array}\right]^{\top}, C_{2}=\left[\begin{array}{l}
1 \\
1 \\
0
\end{array}\right]^{\top}, C_{3}=\left[\begin{array}{l}
1 \\
0 \\
1
\end{array}\right]^{\top} .
\end{gathered}
$$

El sistema descrito cuenta con tres estados. El primero de ellos, $x_{1}$ es un estado con dinámica inestable que se considera completamente desacoplado de los otros dos. Por otro lado, el segundo y tercer estados $\left(x_{2}\right.$ y $\left.x_{3}\right)$ son una pareja de polos conjugados con una dinámica críticamente estable.

Como se puede observar y se ha supuesto durante todo el documento las parejas $\left(C_{2}, A\right)$ y $\left(C_{3}, A\right)$ son observables y se han diseñado las matrices $L_{2}$ y $L_{3}$ de forma adecuada para garantizar la estabilidad del observador. En cuanto al Agente 1, es capaz de observar el primer estado a través de medidas locales mientras que los otros dos estados los estima a partir de la estimación hecha por sus vecinos. De esta forma, las siguientes matrices pueden ser construidas:

$$
V_{1}=\left[\begin{array}{l}
1 \\
0 \\
0
\end{array}\right], \quad \bar{V}_{1}=\left[\begin{array}{ll}
0 & 0 \\
1 & 0 \\
0 & 1
\end{array}\right]
$$

Considerando estas matrices $V_{1}$ y $\bar{V}_{1}$, la matriz del sistema $A$ y un valor mínimo para $c_{1,2}$ y $c_{1,3}$ de $C_{\text {min }}=0.1$, se pueden obtener las siguientes ganancias $N_{1,2}$ y $N_{1,3}$ :

$$
N_{1,2}=N_{1,3}=\left[\begin{array}{cc}
0.0977 & -0.0438 \\
0.0624 & 0.0472
\end{array}\right] \text {. }
$$

Así, los autovalores de las matrices $M_{1}, M_{2}, M_{3}$ y $M_{4}$ pertenecerán al círculo unidad y por consiguiente, garantizarán el diseño robusto del observador (observe la Figura 2).

Ejemplo 1. Supongamos un escenario en régimen permanente en el que la estimación hecha por todos los agentes coincide con el estado de la planta. En ese momento, el sensor del Agente 2 comienza a fallar, repercutiendo en su correcta estimación del estado de la planta. Este fenómeno será simulado mediante la suma de un ruido blanco Gaussiano a la evolución de la estimación del estado de este agente. Fijamos un valor de $r_{1,2}=r_{1,3}=0.1$ 


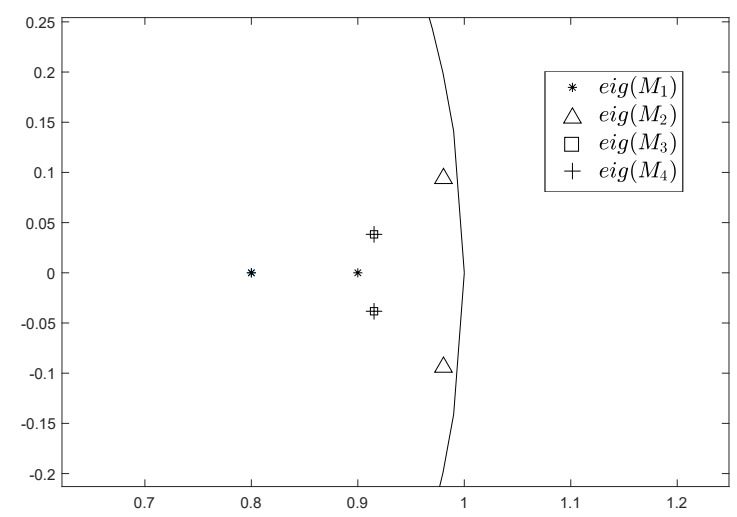

Figura 2: Autovalores de las matrices $M_{1}, M_{2}, M_{3}$ y $M_{4}$ representados en el círculo unidad.
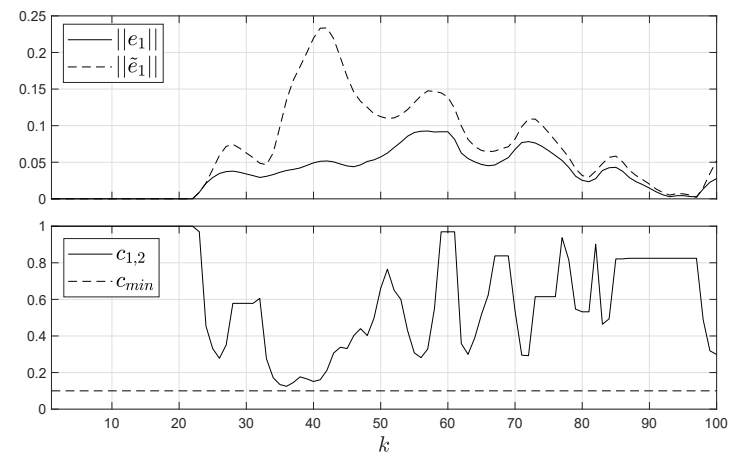

Figura 3: Evolución de la norma del error de estimación del Agente 1 cuando se aplica el algoritmo descrito en este artículo y cuando el peso $c_{1,2}$ se mantiene constante (en líneas discontinuas).

y consideraremos $Q=I_{n \bar{o}_{1}}$. De este modo, la distancia $d_{Q}\left(e_{1,2}\right)$ aumente conforme más cuando el Agente 2 proporciona información incorrecta, haciéndole entrando en la zona de alerta $\mathcal{A}$, y variando el Agente 1 conseguentemente el peso $c_{1,2}$ de acuerdo a lo expuesto en la Sección 5.1.3.

En la Figura 3 se muestra la evolución de la norma del error de estimación del Agente 1 en cada instante de tiempo para dos casos: cuando se modifican los pesos $c_{1,2}$ de acuerdo a lo descrito en la Sección 5.1.3 y el caso en el que se mantienen fijos en un valor $c_{1,2}=1$. Además, se presentan los diferentes valores del peso $c_{1,2}$ obtenidos tras aplicar el algoritmo.

Como se puede observar, el algoritmo descrito introduce una mejora considerable en el desempeño del estimador reduciendo el error de estimación en un alto porcentaje.

Ejemplo 2. Partiendo de las mismas condiciones que en el ejemplo anterior, supongamos que el Agente 2 comienza a sufrir alteraciones en la estimación del estado de la planta que simularemos como un ruido de cada vez mayor energía. Este ruido aumentará hasta el momento en el que el Agente 1 decida no continuar considerándolo para la estimación que realiza.

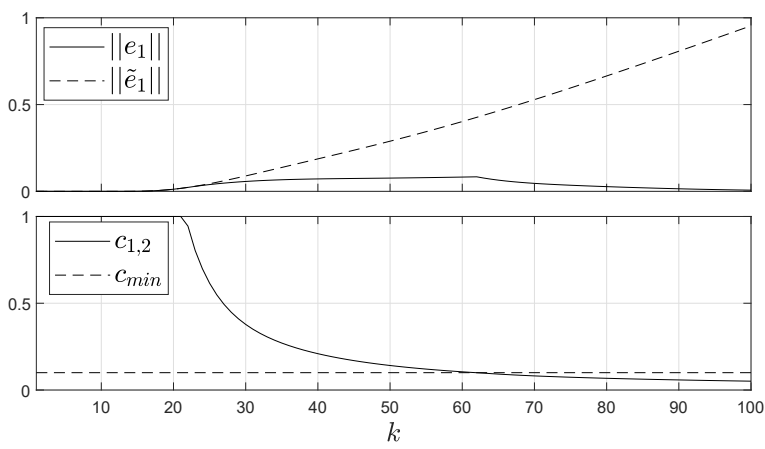

Figura 4: Evolución de la norma del error de estimación del Agente 1 cuando se aplica el algoritmo descrito en este artículo y cuando el peso $c_{1,2}$ se mantiene constante (en líneas discontinuas).

En la Figura 4 se observa cómo el valor de $c_{1,2}$ va disminuyendo hasta sobrepasar $c_{\text {min }}$, en ese momento, la estructura de observador deja de considerar la información del Agente 2 y lleva a cabo un nuevo diseño de $N_{1,2}$. A partir de ese momento, el vecindario del Agente 1 se reduce al Agente 3. Como se puede observar, en esta situación sigue cumpliéndose la hipótesis de observabilidad colectiva realizada. Así, el error de estimación recupera su convergencia asintótica a cero.

\section{Conclusiones}

Este artículo ha mostrado una novedosa forma de abordar el problema de estimación distribuida del estado de una planta por un conjunto de agentes cuando se consideran entidades maliciosas. A partir del concepto de confianza es posible cuantificar la veracidad de la información suministrada por los agentes y, por consiguiente, minimizar el efecto de ruidos y perturbaciones en la estimación de estado.

Se ha mostrado en simulación las mejoras conseguidas para una red formada por tres agentes. Como futuras líneas de trabajo se plantea la generalización del ejemplo presentado en este artículo a un número indeterminado de agentes.

\section{Agradecimientos}

Trabajo parcialmente financiado por AEI/FEDER (TEC2016-80242-P) a través del Laboratorio de Simulación Hardware-in-the-loop de Sistemas Ciberfísicos (LaSSiC).

\section{English summary}

TRUST-BASED DISTRIBUTED 


\section{STATE ESTIMATION OF LTI SYSTEMS}

\begin{abstract}
This paper deals with the problem of distributedly estimate the state of a plant by a network of interconnected agents. The main aim of each agent is to reconstruct the whole state based on their local measurements and the information exchanged with the neighboring agents. We consider the existence of random events such as cyber-attacks or link failures that can affect to the agents and spread through the network. The paper introduces a robust design method and an algorithm in order to face these problems.
\end{abstract}

Keywords: Distributed estimation. LTI systems. Cyber-attacks.

\section{Referencias}

[1] A. R. del Nozal, P. Millán, L. Orihuela, A. Seuret, and L. Zaccarian. Distributed estimation based on multi-hop subspace decomposition. Automatica, 99:213 - 220, 2019.

[2] A. R. del Nozal et al. Diseño LQ e implementación distribuida para la estimación de estado. In XXXVIII Jornadas de Automatica Gijón, September 2017.

[3] Gilles L. et al. Distributed kalman filtering compared to fourier domain preconditioned conjugate gradient for laser guide star tomography on extremely large telescopes. Journal of the Optical Society of America, 30(5):898909, 2013.

[4] M. Farina, G. Ferrari-Trecate, and R. Scattolini. Distributed moving horizon estimation for sensor networks. In 1st IFAC Workshop on Estimation and Control of Networked Systems, pages 126-131, Venice, Italy, 2009.

[5] J. Hespanha, P Naghshtabrizi, and Y. Xu. A survey of recent results in networked control systems. Proceedings of the IEEE, 95(1):138162, 2007.

[6] I. Matei, J. S. Baras, and V. Srinivasan. Trust-based multi-agent filtering for increased smart grid security. In 2012 20th Mediterranean Conference on Control Automation (MED), pages 716-721, July 2012.

[7] P. Millán, A. R. del Nozal, L. Zaccarian, L. Orihuela, and A. Seuret. Distributed implementation and design for state estimation. In 20th IFAC World Congress, volume 50, pages $6483-6488$, Toulouse, July 2017.

[8] Pablo Millán, Luis Orihuela, Carlos Vivas, and Francisco R Rubio. Distributed consensus-based estimation considering network induced delays and dropouts. Automatica, 48(10):2726-2729, 2012.

[9] Hanly S.V. Ng B.L., Evans J.S. and Aktas D. Distributed dowlink beamforming with cooperative base stations. IEEE Transactions on Information Theory, 54(12):5491-5499, 2008.

[10] R. Olfati-Saber. Distributed Kalman filtering for sensor networks. In Proceedings of the IEEE Conference on Decision and Control $(C D C)$, pages 5492-5498, LA, December 2007.

[11] R. Olfati-Saber. Kalman-consensus filter: Optimality, stability, and performance. In 48th IEEE Conference on Decision and Control and 28th Chinese Control Conference, pages 7036-7042, Shanghai, P.R. China, December 2009.

[12] A. H. Sayed. Adaptive networks. Proceedings of the IEEE, 102(4):460-497, 2014.

[13] Chen S.Y. Kalman filter for robot vision: a survey. IEEE Transactions on Industrial Electronics, 59(11):4409-4420, 2012.

[14] Lin Xiao, Stephen Boyd, and Sanjay Lall. A scheme for robust distributed sensor fusion based on average consensus. In IPSN 2005. Fourth International Symposium on Information Processing in Sensor Networks, 2005., pages 63-70. IEEE, 2005.

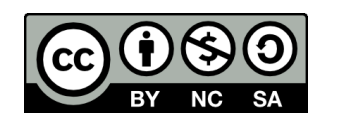

(C) 2019 by the authors. Submitted for possible open access publication under the terms and conditions of the Creative Commons Attribution CC BY-NC-SA 4.0 license (https://creativecommons.org/licenses/by-ncsa/4.0/deed.es). 